\title{
CAPÍTULO 23: A INFLUÊNCIA DOS MÉTODOS DE EXTRAÇÃO NAS CARACTERÍSTICAS FÍSICO-QUÍMICAS DAS BEBIDAS DE CAFÉ: UMA REVISÃO
}

\author{
CAPÍTULO 23: LA INFLUENCIA DE LOS MÉTODOS DE EXTRACCIÓN EN LAS \\ CARACTERÍSTICS FÍSICO-QUÍMICAS DE LAS BEBIDAS DE CAFÉ: UNA \\ REVISIÓN
}

\section{CHAPTER 23: THE INFLUENCE OF EXTRACTION METHODS ON PHYSICO- CHEMICAL CHARACTERISTICS OF COFFEE BERAVAGES: A REVIEW}

\author{
Wallysson Wagner Vilela Santos ${ }^{1}$; Ana Letícia Toté Medeiros ${ }^{2}$; Maria Vitória Lima Costa Donato ${ }^{3}$, Gustavo \\ Henrique Daniel Santos Silva ${ }^{4}$; Suzana Pedroza da Silva ${ }^{5}$
}

DOI: https://doi.org/10.31692/978-65-88970-18-8.329-343

\begin{abstract}
RESUMO
O café está entre as bebidas mais consumidas no mundo e encontra-se em segundo lugar entre as commodities mais comercializadas mundialmente. Após o processo de torrefação e moagem dos grãos, é possível extrair a bebida café, devido a transferência de massa de sólidos solúveis presentes no pó para a água quente ou fria. Existem diversas formas de extrair a bebida de café (decocção, infusão, pressão, percolação e filtração). A escolha de como será preparada a bebida pode influenciar nos principais atributos de qualidade durante a degustação, tendo em vista que a bebida possui complexidade sensorial e em sua composição química. Este trabalho teve como objetivo realizar um minireview em relação a algumas características físicas e químicas da bebida café preparadas pelos métodos de extração filtrados conhecidos como Aeropress, Clever, V60 e Koar. Utilizou-se as principais plataformas de buscas como SciELO, Google Scholar, Portal de Periódicos CAPES, Springer e Science Direct para a coleta dos artigos relacionados aos métodos de extração e caracterização físico-química da bebida de café. Utilizaram-se as seguintes palavras-chave: Koar; Hario V60; Clever Dripp Coffee; Aeropress; Métodos de extração de café; Extraction coffee beverage; Extraction methods. Foram encontrados 23 artigos, entretanto apenas 7 destes foram selecionados para obtenção das informações mais robustas. Os dados referentes a caracterização físico-química das bebidas de café preparadas a partir de grãos da espécie Coffea arabica, pelos diferentes métodos de extração foram: pH, Sólidos Solúveis Totais, Cafeína, Ácido Clorogênico e Lipídeos. Os valores de pH diminuem para os métodos com menor tempo de solubilização dos ácidos orgânicos. O maior tempo de infusão aumenta a concentração dos sólidos solúveis totais, de cafeína e de ácidos clorogênicos presentes na bebida. Para o teor de lipídeos não foi possível se realizar uma comparação pela pouca quantidade de estudos realizados entre os métodos. Não foram encontrados artigos que tratassem do método de extração conhecido como Koar. Conclui-se que o tipo de extração dos filtrados com ou sem escoamento, acrescentados de infusão e/ou pressão levaram a alterações na composição química e características físico-químicas de suas bebidas, reforçando assim, a necessidade de serem realizados mais estudos quanto as especificidades dos métodos para melhor qualidade final da bebida.
\end{abstract}

Palavras-Chave: Café, Características físico-químicas, Métodos de extração.

\section{RESUMEN}

El café se encuentra entre las bebidas más consumidas en el mundo y ocupa el segundo lugar entre los productos básicos más comercializados a nivel mundial. Después del proceso de tostado y molido de los granos, es posible extraer la bebida de café, debido a la transferencia de masa de sólidos solubles

\footnotetext{
${ }^{1}$ Engenharia de Alimentos, Universidade Federal do Agreste de Pernambuco, wallysson70@gmail.com

${ }^{2}$ Engenharia de Alimentos, Universidade Federal do Agreste de Pernambuco, analeticiatote@gmail.com

${ }^{3}$ Engenharia de Alimentos, Universidade Federal do Agreste de Pernambuco, vitoriadonato57@gmail.com

${ }^{4}$ Engenharia de Alimentos, Universidade Federal do Agreste de Pernambuco, gd30440@gmail.com

${ }^{5}$ Doutora em Engenharia Química, Universidade Federal do Agreste de Pernambuco,

suzana.pedroza@ufape.edu.br
} 
presentes en el polvo al agua fría o caliente. Hay varias formas de extraer la bebida de café (decocción, infusión, presión, percolación y filtración). La elección de cómo se preparará la bebida puede influir en los principales atributos de calidad durante la degustación, considerando que la bebida tiene complejidad sensorial y su composición química. Este trabajo tuvo como objetivo realizar una minirrevisión en relación a algunas características físicas y químicas de la bebida de café elaborada por los métodos de extracción por filtración conocidos como Aeropress, Clever, V60 y Koar. Se utilizaron las principales plataformas de búsqueda como SciELO, Google Scholar, CAPES Journal Portal, Springer y Science Direct para recolectar artículos relacionados con la extracción y caracterización físico-química de la bebida de café. Se utilizaron las siguientes palabras clave: Koar; Hario V60; Café inteligente por goteo; Aeropress; Métodos de extracción de café; Bebida de extracción de café; Métodos de extracción. Se encontraron 23 artículos, sin embargo, solo se seleccionaron 7 de estos para obtener la información más sólida. Los datos referentes a la caracterización físico-química de bebidas de café elaboradas a partir de granos de la especie Coffea arabica, por los diferentes métodos de extracción fueron: $\mathrm{pH}$, Sólidos Solubles Totales, Cafeína, Ácido Clorogénico y Lípidos. Los valores de pH disminuyen para los métodos con un tiempo de solubilización más corto para los ácidos orgánicos. El tiempo de infusión más largo aumenta la concentración de sólidos solubles totales, cafeína y ácidos clorogénicos presentes en la bebida. Para el contenido de lípidos, no fue posible hacer una comparación debido a la pequeña cantidad de estudios realizados entre los métodos. No se encontraron artículos que trataran sobre el método de extracción conocido como Koar. Se concluye que el tipo de extracción de los filtrados con o sin flujo, adicionados con infusión y / o presión, provocó cambios en la composición química y características físico-químicas de sus bebidas, reforzando así la necesidad de más estudios sobre las especificidades de los métodos para una mejor calidad final de la bebida.

Palabras Clave: Café, Características fisicoquímicas, Métodos de extracción.

\section{ABSTRACT}

Coffee is among the most consumed beverages in the world and ranks second among the most traded commodities worldwide. After the process of roasting and grinding the beans, it is possible to extract the coffee drink, due to the mass transfer of soluble solids present in the powder to the hot or cold water. There are several ways to extract the coffee drink (decoction, infusion, pressure, percolation and filtration). The choice of how the drink will be prepared can influence the main quality attributes during tasting, considering that the drink has sensory complexity and its chemical composition. This work aimed to carry out a minireview in relation to some physical and chemical characteristics of the coffee drink prepared by the filtered extraction methods known as Aeropress, Clever, V60 and Koar. The main search platforms such as SciELO, Google Scholar, CAPES Journal Portal, Springer and Science Direct were used to collect articles related to the extraction and physical-chemical characterization of the coffee drink. The following keywords were used: Koar; Hario V60; Clever Dripp Coffee; Aeropress; Coffee extraction methods; Extraction coffee beverage; Extraction methods. 23 articles were found, however only 7 of these were selected to obtain the most robust information. The data referring to the physicalchemical characterization of coffee drinks prepared from grains of the species Coffea arabica, by the different extraction methods were: $\mathrm{pH}$, Total Soluble Solids, Caffeine, Chlorogenic Acid and Lipids. The $\mathrm{pH}$ values decrease for methods with shorter solubilization time for organic acids. The longer infusion time increases the concentration of total soluble solids, caffeine and chlorogenic acids present in the drink. For the lipid content, it was not possible to make a comparison due to the small amount of studies carried out between the methods. No articles were found that dealt with the extraction method known as Koar. It is concluded that the type of extraction of the filtrates with or without flow, added with infusion and / or pressure led to changes in the chemical composition and physical-chemical characteristics of their drinks, thus reinforcing the need for further studies on the specificities of the methods for better final quality of the drink.

Keywords: Coffee, Physico-chemical characteristics, Extraction methods. 
Segunda commodity mais comercializada no mundo, o café também é considerado uma das bebidas do tipo não alcoólicas mais consumidas (HAILE; KANG, 2019; SALES et al., 2020). No Brasil, a cafeicultura possui grande importância para a economia, segundo a Companhia Nacional de Abastecimento (CONAB, 2020a) o país é o maior produtor e exportador de café do mundo. Em 2020, o país teve produção estimada em 63,08 milhões de sacas de café beneficiadas, e obteve um aumento de $27,90 \%$ comparado com a produção da safra de 2019 (CONAB, 2020b).

O café é uma bebida bastante complexa em termos de constituição química e sensorial (FERRÃO et al., 2017) e pode ser consumida em diferentes formas, a depender das preferências pessoais, comportamentos sociais, culturais, entre outros fatores (CAPORASO et al., 2014). A escolha do método de extração associada a outras variáveis como tempo de extração, temperatura, proporção café/água, entre outros, são capazes de influenciar os atributos que são determinantes para qualidade do café na xícara (MORONEY et al., 2015; CÓRDOBA et al., 2020; SANTOS et al., 2020).

Segundo Angeloni et al. (2019), a extração da bebida de café é obtida a partir do café torrado e moído, e podem ser utilizadas diferentes formas de preparo, que se baseiam em um processo de extração do tipo sólido-líquido, ou seja, ocorre a absorção de água pelo café moído, a transferência em massa de sólidos solúveis presentes no pó para a água quente e a separação do extrato resultante dos sólidos.

Neste trabalho foram abordados os seguintes métodos de extração: Aeropress, Clever, V60 e Koar. O presente estudo teve como objetivo determinar uma relação entre a composição química e físico-química na bebida café extraída em diferentes métodos de extração a partir de levantamento bibliográfico.

\section{REFERENCIAL TEÓRICO}

Na literatura é possível encontrar diversos estudos (PEREIRA et al., 2019; ZAPATA; ARANGO; ROJANO, 2019a; ZAPATA; ARANGO; ROJANO, 2019b; SUNARHARUM; FARHAN, 2020) que relacionam a composição da bebida com as características sensoriais e as preferências de consumo com as formas que as bebidas são extraídas. Segundo Santos et al. (2020), a escolha dos métodos de extração pode influenciar nos parâmetros considerados determinantes no que se refere à qualidade da bebida. 
A extração da bebida de café torna-se possível devido ao processo de torrefação e moagem dos grãos, após estas etapas, os componentes solúveis do café podem ser extraídos em água quente, resultando na bebida apreciada por milhares de consumidores no mundo todo. Existem diversas formas de extrair o café, e estas podem ser agrupados por categorias como: decocção, infusão, pressão, percolação e filtragem (MORONEY et al, 2015; PETRACCO, 2001). Neste estudo serão abordados métodos de extração que utilizam os sistemas de pressão, filtragem e infusão, ou combinação destes.

Criado pela empresa Aerobie ${ }^{\circ}$ em 2005, o Aeropress (Figura 01) é composto por uma borracha de vedação, um porta filtro e dois cilindros de plástico que agem em conjunto, como um sistema de uma seringa, fazendo a introdução do ar e a extração é conduzida com a aplicação da pressão (ZAPATA; ARANGO E ROJANO, 2019b).

Figura 01: Características físicas do método de extração Aeropress.

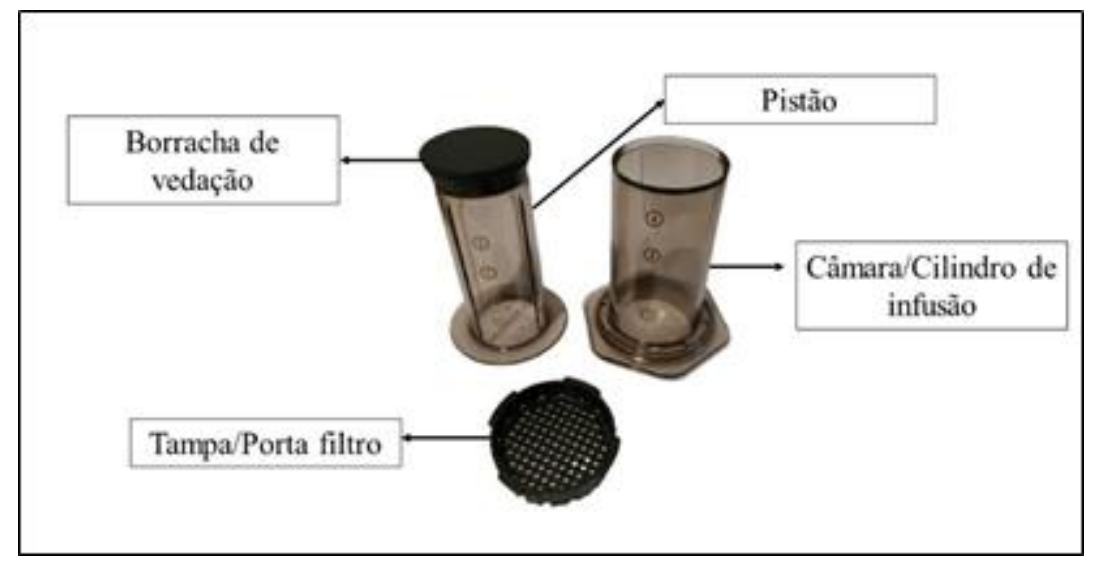

Fonte: Própria, 2021.

Este equipamento basicamente funciona a partir da combinação dos métodos que fazem o uso da infusão e filtragem com aplicação da pressão manual feita durante a extração da bebida. O Aeropress é conhecido por resultar em uma bebida que sensorialmente possui características que se assemelham com o café espresso e filtrado (TAVARES; MOURAD, 2020). A Figura 02 detalha as etapas do processo de extração da bebida no método Aeropress. 


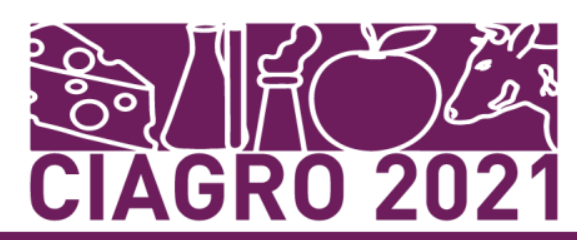

Figura 02: Processo de extração da bebida no método Aeropress.

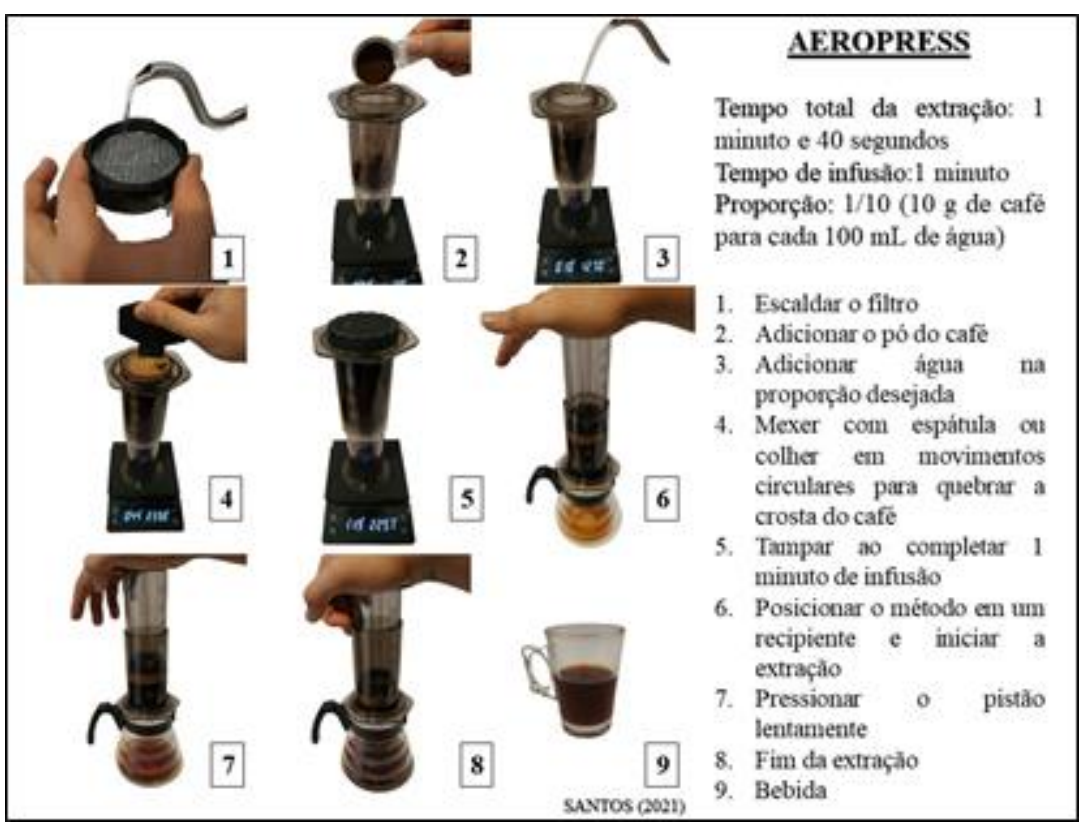

Fonte: Própria, 2021.

A Clever (Figura 03) é bastante similar aos coadores que usam filtros de papel em formato cônico. Este método foi desenvolvido em meados dos anos 2000 pela empresa Taiwanesa Absolutely Best Idea Delevolpment ${ }^{\circledR}$, que tiveram como inspiração o sistema de filtragem comum que utiliza os filtros de papel. Uma das diferenças do funcionamento da Clever, é que o café fica em infusão até o momento da extração, resultando em bebidas com características dos métodos de infusão e gravidade (percolação) (EASTO; WILLHOFF, 2017; GURGEL; RELVAS, 2018).

Figura 03: Características físicas do método de extração Clever.

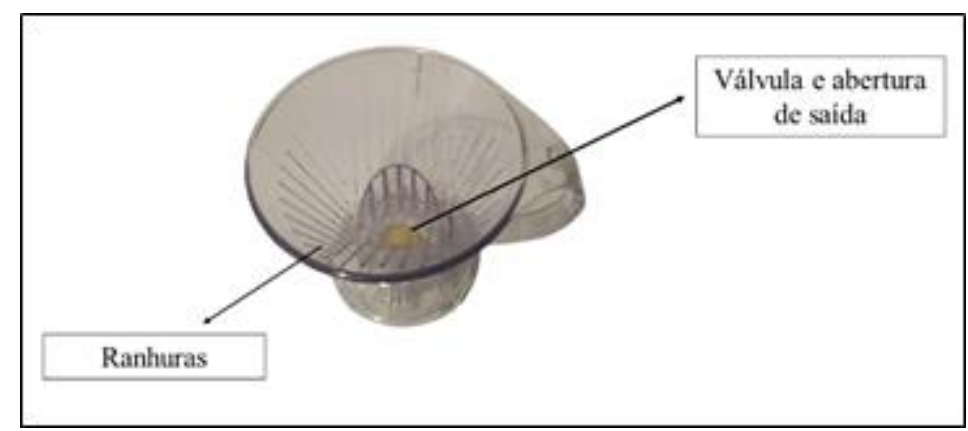

Fonte: Própria, 2021.

Devido seu sistema de funcionamento, a Clever é praticamente uma junção da Prensa Francesa com o Coador de Papel. Por possuir uma válvula de saída localizada no interior do porta filtro, a Clever possibilita maior controle do tempo em infusão, uma vez que o método 
quando posicionado em cima de algum recipiente, faz com que esta válvula libere automaticamente o fluxo (bebida de café) e finaliza a extração da bebida (ESPRESSO, 2016; EASTO; WILLHOFF, 2017). A Figura 04 elucida as etapas de extração da bebida utilizando este método de extração.

Figura 04: Processo de extração da bebida no método Clever.

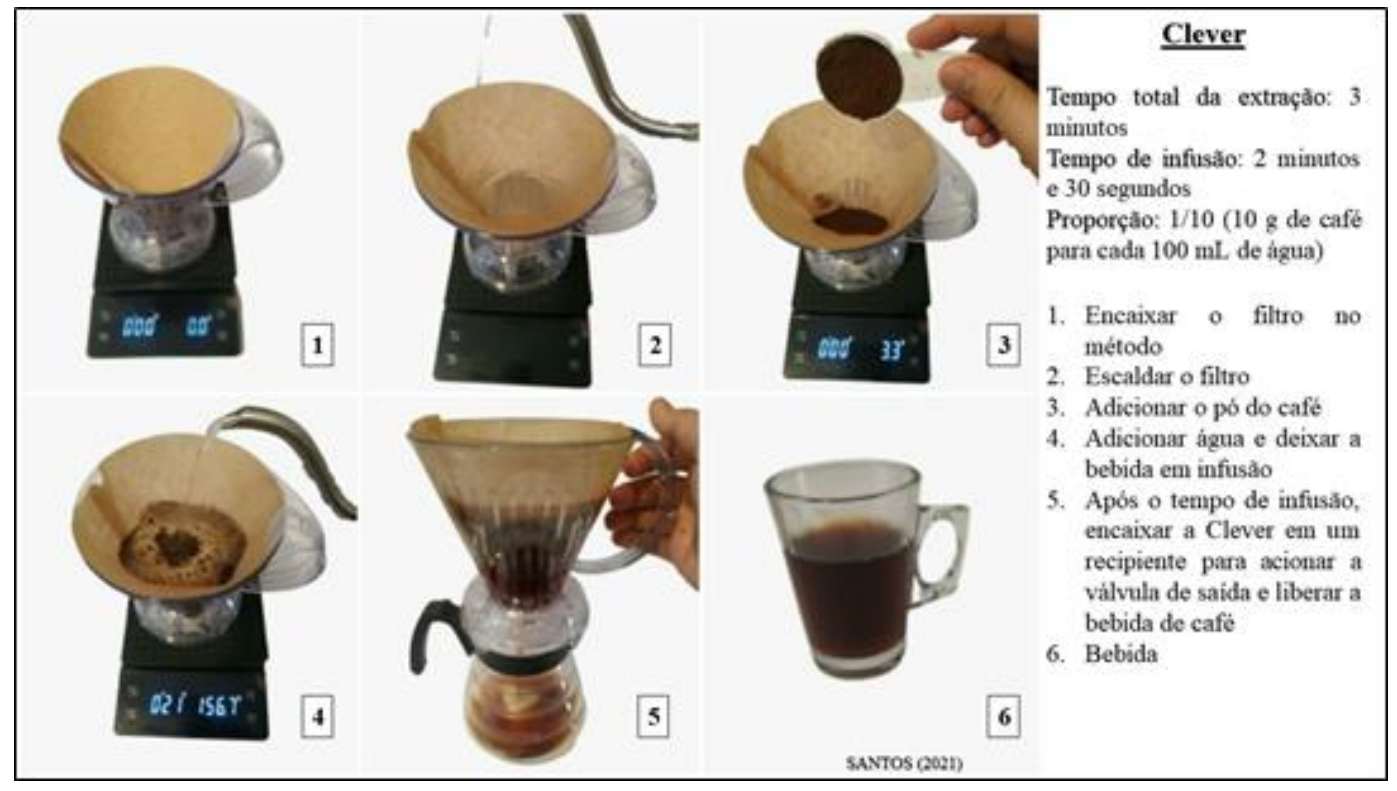

Fonte: Própria, 2021.

Devido angulação e formato, este método de extração é bastante conhecido como "Hario V60" ou apenas como V60 (Figura 05), é composto por um porta filtro (criado por uma empresa japonesa Hario® em 2005) e apresenta formato cônico com angulação de $60^{\circ}$ (EASTO; WILLHOFF, 2017).

Figura 05: Características físicas do método de extração V60.

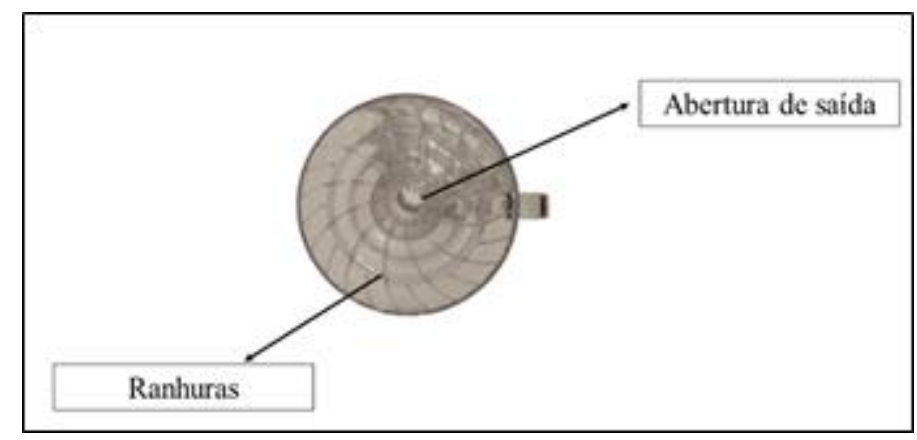

Fonte: Própria, 2021. 
A abertura de saída do V60 é maior quando comparada com outros métodos que funcionam por filtração, o que facilita a vazão da bebida durante a extração, além de apresentar ranhuras espiraladas em suas paredes que contribuem para a expansão do café (EASTO; WILLHOFF, 2017; GURGEL; RELVAS, 2018). A Figura 06 elucida as etapas de extração para obtenção da bebida de café neste método.

Figura 06: Processo de extração da bebida no método V60.

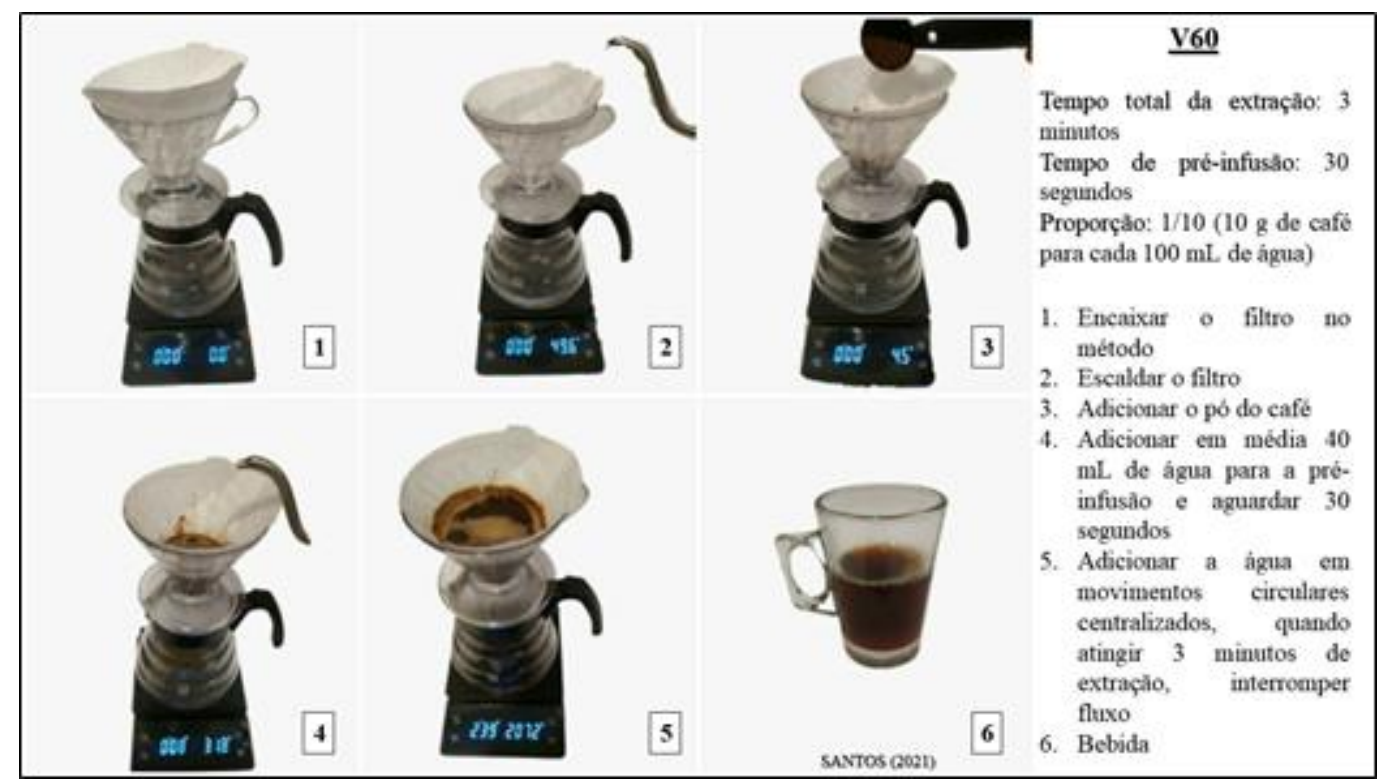

Fonte: Própria, 2021.

Desenvolvido em Pernambuco no ano de 2017, o Koar (Figura 07) é um método que tem como proposta realçar a doçura, corpo e equilibrar a acidez da bebida (ESPRESSO, 2018). Este método possui 16 sulcos oblíquos com angulação de $55^{\circ}$ em forma cônica e apresenta uma abertura para o escoamento da bebida durante a extração de 16 mm (KOAR, 2020).

Figura 07: Características físicas do método Koar.

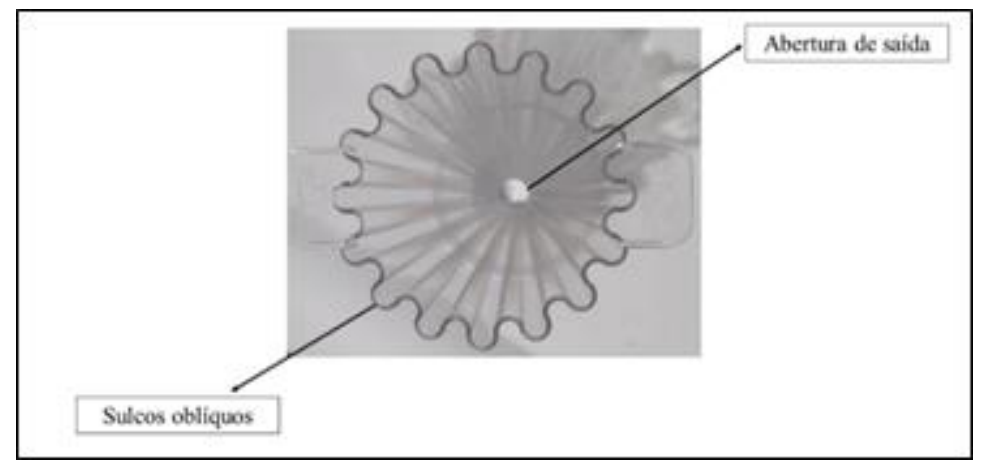

Fonte: Própria, 2021. 
De acordo com o fabricante (KOAR, 2020), a inclinação e a profundidade dos sulcos

são as características físicas responsáveis pelo escoamento rápido da água, isto acontece, pois, o fluido quando está em movimento dentro dos sulcos se comprime. E quando encontra uma zona de estreitamento, ocorre a diminuição da sua pressão e, portanto, aumento da sua velocidade, permitindo uma extração mais rápida. Além disso, os sulcos também permitem o afastamento do filtro de papel das paredes do método, que também influencia no momento do preparo, consequentemente no tempo de extração da bebida. A Figura 08 elucida as etapas de extração da bebida.

Figura 08: Processo de extração da bebida no Koar.

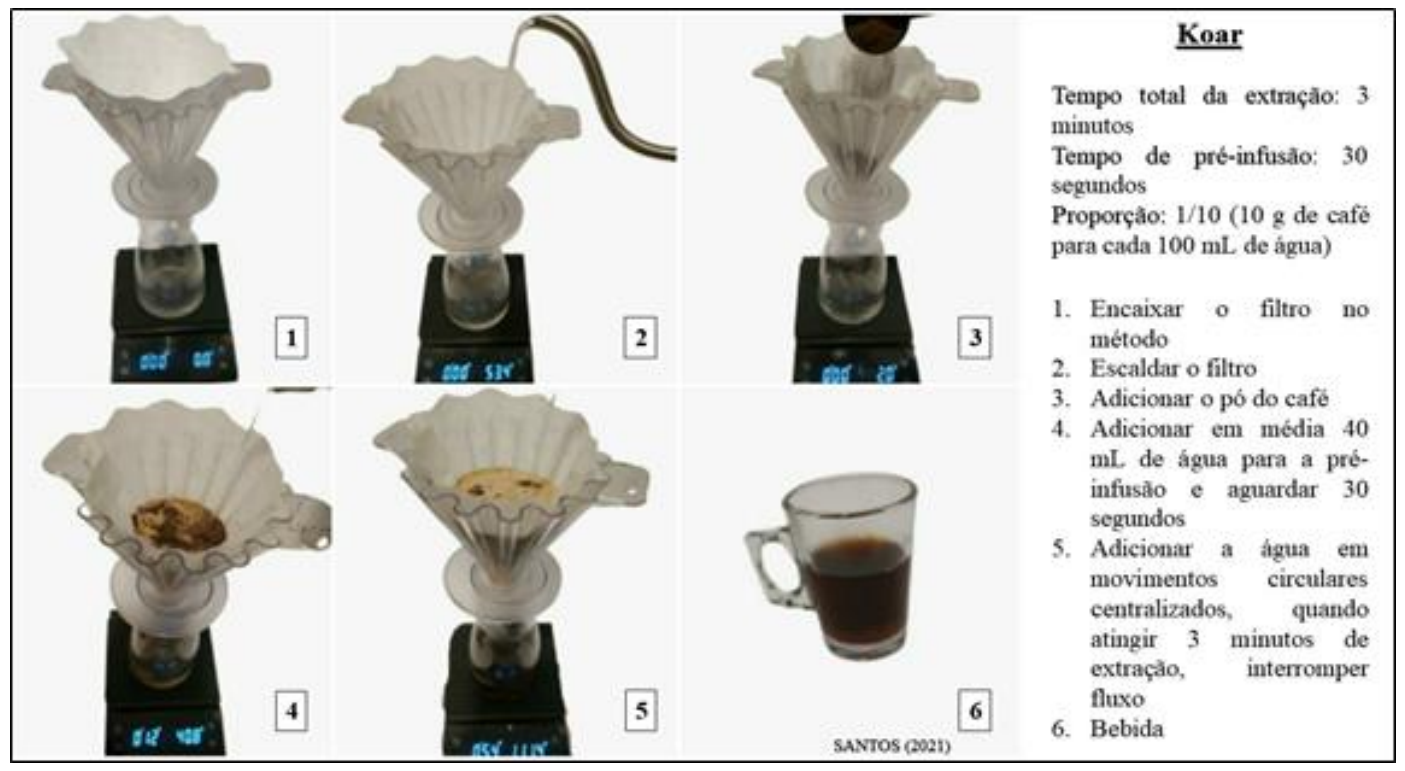

Fonte: Própria, 2021.

\section{METODOLOGIA}

Para a realização deste minireview foram utilizadas as principais plataformas de buscas como SciELO (Scientific Electronic Library Online), Google Scholar, Portal de Periódicos CAPES (Coordenação de Aperfeiçoamento de Pessoal de Nível Superior), Springer e Science Direct para a coleta dos artigos relacionados aos métodos de extração e caracterização físicoquímica da bebida de café. Utilizou-se as seguintes palavras-chave: Koar; Hario V60; Clever Dripp Coffee; Aeropress; Métodos de extração de café; Extraction coffee beverage; Extraction methods.

Após esta etapa foram selecionados artigos que tivessem trabalhado com os métodos de preparo filtrados conhecidos como Aeropress, Clever, V60 e Koar; os quais utilizam a extração 
por pressão, infusão e/ou percolação, como já descrito na fundamentação teórica. É importante ressaltar que os artigos selecionados são publicações de relevância, o que indica a qualidade dos trabalhos realizados pelos autores (Tabela 01).

Os dados encontrados na literatura, referentes à caracterização físico-química das bebidas de café, foram obtidos através de análises em grãos da espécie Coffea arabica por diferentes métodos de extração. Os parâmetros de pH, Sólidos Solúveis Totais (SST) em ${ }^{\circ}$ Brix, Cafeína (mg/mL), Ácido Clorogênico (mg/100 g amostra) e Lipídeos (\% g/g) dos trabalhos encontrados nas plataformas de pesquisas citadas (Tabela 01).

Salienta-se, portanto, que os dados demonstrados na Tabela 02 estão representados em faixas de valores (intervalos entre os resultados) a fim de se ter conhecimentos dos menores e maiores valores, das respectivas análises, encontrados nos artigos selecionados na literatura.

\section{RESULTADOS E DISCUSSÃO}

Com base nas plataformas de pesquisas, foram encontrados um total de 23 artigos, após leitura minuciosa, apenas 7 artigos foram selecionados (Tabela 01), pois os autores utilizaram os mesmos métodos de extração que são objeto de estudo deste trabalho e ainda realizaram análises físico-químicas das bebidas.

Tabela 01: Artigos selecionados na literatura, contendo os métodos de extração propostos (Aeropress, Clever, V60 e Koar) e resultados de análises físico-químicas da bebida café com grãos Coffea arabica.

\begin{tabular}{|c|c|}
\hline Autores (ano) & Método de Extração ${ }^{1}$ \\
\hline Zapata, Arango e Rojano (2019a) & Clever e V60² \\
\hline Tan et al. (2020) & Clever \\
\hline Angeloni et al. (2019) & Aeropress e V60² \\
\hline Zapata, Arango e Rojano (2019b) & Aeropress ${ }^{2}$ \\
\hline Tavares e Mourad (2019) & Aeropress e V60² \\
\hline Sunarharum e Farhan (2020) & Aeropress ${ }^{2}$ \\
\hline Santos e Junior (2018) & $\mathrm{V} 60^{2}$ \\
\hline
\end{tabular}

Para o $\mathrm{pH}$ as faixas de valores encontradas na literatura tiveram valores muito próximos entre si (Tabela 02). Avaliando o tipo de extração utilizada para preparo da bebida (infusão e pressão), Zapata, Arango e Rojano (2019b), afirmam que a pressão aplicada é capaz de realizar a solubilização completa dos ácidos orgânicos presentes no café. O que também explicaria o 
fato do Aeropress ter apresentado o menor valor para o parâmetro de $\mathrm{pH}$ (Tabela 02), tendo em vista a variação encontrada para os outros métodos quanto a este parâmetro.

França, Mendonça e Oliveira (2005), Zapata, Arango e Rojano (2019a) e, Santos et al. (2020) concluíram em seus trabalhos que tanto a qualidade do grão, o tipo de torra e o método de extração podem influenciar no valor do $\mathrm{pH}$ de acordo com a variação da concentração de cafeína, ácido fórmico, ácido acético, trigonelina, ácidos clorogênicos e ácido cítrico presentes. Sendo necessário se manter fixa algumas variáveis para se verificar de fato a influência da infusão ou pressão no valor de pH e composição química dos métodos filtrados.

Tabela 02: Resultados de parâmetros físico-químicos encontrados na literatura para as bebidas bebida café com grãos Coffea arabica extraídas nos métodos de extração Aeropress, Clever, V60 e Koar.

\begin{tabular}{|c|c|c|c|c|c|}
\hline Métodos & $\mathrm{pH}$ & $\begin{array}{c}\text { SST } \\
\left({ }^{\circ} \text { Brix }\right)\end{array}$ & $\begin{array}{l}\text { Cafeína } \\
(\mathrm{mg} / \mathrm{mL})\end{array}$ & $\begin{array}{c}\text { ACG } \\
(\mathrm{mg} / 100 \mathrm{~g} \text { amostra })\end{array}$ & $\begin{array}{l}\text { Lipídeos } \\
(\% \mathrm{~g} / \mathrm{g})\end{array}$ \\
\hline Aeropress & $4,90-5,16$ & $1,30-1,80$ & $0,78^{1}$ & $12,0^{1}$ & $*$ \\
\hline Clever & $5,13^{1}$ & $1,94^{1}$ & $1,05^{1}$ & $0,33-129,0$ & $*$ \\
\hline V60 & $4,92-5,15$ & $1,0-2,0$ & $0,0029-0,74$ & $0,0147-70,0$ & 0,17 \\
\hline Koar & $*$ & $*$ & $*$ & $*$ & $*$ \\
\hline
\end{tabular}

Os Sólidos Solúveis Totais (SST) estão ligados a extração dos compostos químicos presentes no pó do café para água, sendo responsáveis pelo atributo sensorial denominado “corpo da bebida" (EASTO; WILLHOFF, 2017). Nos dados obtidos na literatura, os menores valores de SST obtidos foram para o V60 e o Aeropress o que indica que não passar pela infusão (V60) ou se ter menor tempo de infusão (Aeropress) diminui a concentração dos compostos solúveis na bebida (Tabela 02). Apesar de serem encontrados valores mais altos nas faixas de SST encontradas os artigos selecionados; sendo necessário um mesmo estudo, com maior controle destas variáveis apresentar com maior precisão a influência da infusão para a maior concentração de SST na bebida.

Vale ressaltar que o parâmetro SST pode ser influenciado por diversos fatores, dentre eles, destacam-se: a espécie do café, método de extração, granulometria utilizada, relação água/café, temperatura da água, tempo de extração e níveis de temperaturas empregados no processo de torra dos grãos (SUNARHARUM; FARHAN, 2020; SANTOS et al., 2020).

Observa-se que as bebidas extraídas nos métodos que além de filtração acrescenta-se a 


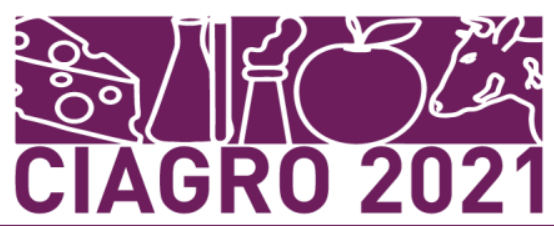

infusão, o Aeropress e a Clever, apresentaram maior retenção de cafeína, sendo um pouco maior para a clever que tem maior tempo de infusão e não utiliza de pressão (Tabela 02). De acordo com Angenoli et al. (2018), diversos fatores podem afetar diretamente o teor de cafeína presente na bebida, inclui-se a temperatura da água durante a extração, a proporção água e café, tipo de moagem (fina, média, grossa), pressão da água, compactação e volume final da bebida. A cafeína é bastante conhecida pelo seu efeito estimulante após o consumo de determinados alimentos e bebidas que a contém além de outros benefícios a saúde em doses diárias controladas (ARAUJO et al., 2020).

Em relação aos ACG há uma maior concentração destes ácidos na bebida quando se tem um maior tempo de infusão (Tabela 02). Porém, o maior valor para a faixa de valores obtida no V60 vai em desencontro ao esperado quando comparado com o método filtrado com um período de infusão acrescentado de pressão (Aeropress), como discutido em Zapata, Arango e Rojano (2019b) e Santos et al. (2020). Sendo necessários estudos mais específicos quanto a cada característica específica de cada método de extração de café.

No estudo de Zapata, Arango e Rojano (2019b) utilizando métodos de extração por filtração por pressão (Aeropress, Cafeteira espresso, Staresso, Presso e Cafeteira Moka Italiana), os autores associaram o teor elevado de ácido clorogênico encontrado nas bebidas de café extraídas em Staresso, Cafeteira espresso e Moka Italiana com o sabor amargo presente nessas bebidas. Santos et al. (2020) em sua pesquisa também relataram maior percentual na bebida preparada na Cafeteira Moka Italiana, que também utiliza de pressão.

Os ácidos clorogênicos desempenham função de grande importância no que se refere ao ponto de vista fisiológico para a planta do café e nos atributos sensoriais como aroma e sabor. Variáveis como: fatores genéticos, espécie e variedade do café, grau de maturação, terroir, tipo de torra utilizada, entre outras, podem afetar a composição dos ácidos clorogênico, consequentemente também afetam a composição final da bebida de café (FARAH; DONANGELO, 2006). Estes ácidos são responsáveis por conferir a maior parte da acidez perceptível nas bebidas, que é um fator desejável no momento de degustar uma xícara de café, e tendem a apresentar uma certa complexidade de sabores da bebida, pois não se trata de um sabor "azedo", mas sim de uma sensação refrescante e frutada ao consumir o café (EASTO; WILLHOFF, 2017).

Quanto ao percentual de lipídios presente na bebida, encontrou-se na literatura apenas valores referentes a este parâmetro para bebida extraída no método V60 (0,17 \% g/g) (Tabela 02). De modo geral, a fração lipídica presente na composição dos alimentos é de suma importância para algumas características como o aroma, textura, estabilidade das proteínas, cor 
Ratnayake et al. (1993) a fim de avaliar o conteúdo lipídico da bebida café preparada por diferentes métodos, observaram que as bebidas extraídas utilizando o filtro de papel apresentaram (de 40 a 125 vezes) menos lipídeos quando comparados com o método bastante conhecido no Brasil como "fervido" (forma de preparo em que o pó do café é adicionado durante a fervura da água, ficando em contato com água até um determinado tempo, não sendo realizado o processo de filtragem da bebida, geralmente espera-se o pó decantar).

Apesar de se ter realizado a busca quanto ao método filtrado conhecido como Koar não foram encontrados resultados, reforçando a necessidade de se conhecer que características físicas e químicas cada método leva a bebida para uma clareza maior do consumidor em relação ao que se está consumindo.

\section{CONSIDERAÇÕES FINAIS}

A partir dos dados obtidos na literatura para os parâmetros pH, Sólidos Solúveis Totais (SST) em ${ }^{\circ}$ Brix, Cafeína (mg/mL), Ácido Clorogênico (mg/100 g amostra) e Lipídeos (\% g/g), entre os métodos filtrados conhecidos como Aeropress, Clever, V60 e Koar foi possível concluir que:

Os valores de $\mathrm{pH}$ foram próximos entre si, demonstrando uma leve diminuição para os métodos filtrados que proporcionam menor tempo de solubilização dos ácidos orgânicos;

Os métodos filtrados que não passam por um período de infusão ou com menor período de infusão diminuem a concentração dos sólidos solúveis totais presentes na bebida final;

Os métodos que além de filtração acrescenta-se a infusão, apresentam maior retenção de cafeína e, que quanto maior este tempo de infusão maior a concentração de caféina presente na bebida;

Quanto aos ácidos clorogênicos há uma maior concentração destes ácidos na bebida quando se tem um maior tempo de infusão proporcionada pelo método;

Para o teor de lipídeos não foi possível se realizar uma comparação pela pouca quantidade de estudos realizados entre os métodos;

Não se encontrou trabalhos na literatura utilizando o método filtrado conhecido como Koar.

Portanto, sugere-se a realização de estudos mais robustos que envolvam as especificidades dos métodos de extração de café e que diferentes características proporcionam na qualidade final da bebida, tendo em vista a escassez de trabalhos abordando essa temática. 


\section{AGRADECIMENTOS}

Agradecemos à Universidade Federal Rural de Pernambuco - UFRPE, Universidade Federal do Agreste de Pernambuco/ Unidade Acadêmica de Garanhuns - UFAPE/ UAG e ao Conselho Nacional de Desenvolvimento Científico e Tecnológico - CNPQ pelo apoio financeiro concedido ao aluno para a realização deste trabalho.

\section{REFERÊNCIAS}

ALVARENGA, S. T. Caracterização química e sensorial de cafés especiais do sul de minas gerais. 120 p. Dissertação (Doutorado em Ciência dos Alimentos) - Universidade Federal de Lavras, Lavras - $\quad$ MG, 2017. Disponível em: http://www.sbicafe.ufv.br/handle/123456789/9109. Acesso em: 08 fev. 2020.

ANGELONI, G.; GUERRINI, L.; MASELlA, P.; BELLUMORI, M.; DALUISO, S.; PARENTI, A.; INNOCENTI, M. What kind of coffee do you drink? An investigation on effects of eight different extraction methods. Food Research International, v. 116, p. 1327 - 1335, 2019. Disponível em: https://doi.org/10.1016/j.foodres.2018.10.022. Acesso em: 25 dez. 2020.

ARAUJO, D. E. P.; DELFINO, F. S.; PROVESI, J. V.; SKIBA, L. G.; HASPER, M. G.; SILVA, P. V.; DIAS, R. C. E. Consumo de cafeína: uma abordagem bioquímica e sociocultural num ambiente escolar. Brazilian Journal of Delevopment, v. 6, n. 7, p. 50071-50089, 2020. Disponível em: https://doi.org/10.34117/bjdv6n7-601Acesso em: 06 fev. 2021.

CAPORASO, N.; GENOVESE, A.; CANELA, M. D.; CIVITELlA, A.; SACCHI, R. Neapolitan coffee brew chemical analysis in comparison to espresso, moka and American brews. Food Research International, v. 61, p. 1-9, 2014. Disponível em: http://dx.doi.org/10.1016/j.foodres.2014.01.020. Acesso em: 29 dez. 2020.

CÓRDOBA, N; FERNANDEZ-ALDUENDA, M. MORENO, F. L; RUIZ, Y. Coffee extraction: A review of parameters and their influence on the physicochemical characteristics and flavour of coffee brews. Trends in Food Science \& Technology, V. 96, p. 45-60, 2020. Disponível em: https://doi.org/10.1016/j.tifs.2019.12.004. Acesso em: 29 dez. 2020.

CONAB - Companhia Nacional de Abastecimento. Acompanhamento da safra brasileira de café: Quarto levantamento. Brasília, v. 6, n.4 p.8, p.45, 2020b. Disponível em:https://www.conab.gov.br/component/k2/item/download/34932_f1feea7816de1bd2f 9528cac2d9a19b1. Acesso em: 20 dez. 2020.

CONAB - Companhia Nacional de Abastecimento. Café: Análise mensal - Junho/Julho 2020. Brasília, p.2-5, p.22, 2020a. Disponível em: https://www.conab.gov.br/info-agro/analises-domercado-agropecuario-e-extrativista/analises-do-mercado/historico-mensal-de-café. Acesso em: 20 dez. 2020. 
EASTO, J.; WILlHOFF, A. Craft Coffee: A Manual: Brewing a Better Cup at Home. First Edition. Chicago: Surrey Books, 2017. 272p. p.65-66, 79-80, 186.

ESPRESSO - café, gastronomia e viver. Café \& preparos. 2016. Disponível em: https://revistaespresso.com.br/2016/06/29/filtro-pratico/. Acesso em: 19 jan. 2021.

ESPRESSO - café, gastronomia e viver. Método com referências da cultura pernambucana chega ao mercado. 2018. Disponível em: https://revistaespresso.com.br/2018/06/06/metodocom-referencias-da-cultura-pernambucana-chega-ao-mercado/. Acesso em: 04 jan. 2021.

FARAH, A.; DONANGELO, C. M. Phenolic compounds in coffee. Brazilian Journal of Plant Physiology, v. 18, n. 1, p. 23-36, 2006. Disponível em: https://doi.org/10.1590/S167704202006000100003. Acesso em: 08 fev. 2021.

FERRÃO, R. G; FONSECA, A. F. A; FERRÃO, M. A. G; MUNER, L. H. Coffea Canephora. In: Conilon Coffee. $2^{a}$ Edição. Vitória, ES: Incaper, 2017. 786p. p. 37- 50. Disponível em: http://portalcoffea.com/wp-content/uploads/2018/11/Livro-Cafe-Conilon-2a-Edicao.pdf. Acesso em: 20 dez. 2020.

FRANCA, A. S; MENDONÇA, J. C. F.; OLIVEIRA, S. D. Composition of green and roasted coffees of different cup qualities. Food Science and Technology, v. 38, n. 7, p. 709-715.

GURGEL, M.; RELVAS, E. Café com design: A arte de beber café. $2^{a}$ edição, São Paulo: Senac São Paulo, 2018. 215p. p. p. 42, 47-48.

HAILE, M.; KANG, W. H. The role of microbes in coffee fermentation and their impact on coffee quality. Journal of Food Quality, v. 2019, p.1-6, 2019. Disponível em: https://doi.org/10.1155/2019/4836709. Acesso em: 20 dez. 2020.

KOAR. Koar. 2020. Disponível em: https://www.koar.com.br/koar/. Acesso em: 04 jan. 2021.

MORONEY, K. M.; LEE, W. T; O'BRIEN, S. B. G.; SUIJVER, F.; MARRA J. Modelling of coffee extraction during brewing using multiscale methods: An experimentally validated model. Chemical Engineering Science. v. 137 p. 216-234, 2015. Disponível em: https://doi.org/10.1016/j.ces.2015.06.003. Acesso em 29 dez. 2020.

PEREIRA, L. L.; MORELI, A. P.; JUNIOR, D. B.; SOUSA, L. H. B. P.; MARCATE, J. P. P.; OLIVEIRO, G. F.; DEBONA, D. G.; GUARÇONI, R. C. Construção de perfil sensorial para o café conilon fermentado. IfesCiência, v. 5, n.1, p. 242-252, 2019. Disponível em: https://doi.org/10.36524/ricv5i2.461. Acesso em: 21 dez. 2020.

PETRACCO, M. Technology IV: Beverage preparation: Brewing Trends for the New Millennium. In: CLARKE, R. J.; VITZTHUM, O. G. Coffee: Recent Developments. Oxford, United Kingdom: Blackwell Science, 2001, 257p. p 140-164. Disponível em: https://doi.org/10.1002/9780470690499.ch7. Acesso em: 09 jan. 2021.

RATNAYAKE, W. M. N.; HOLLYWOOD, R.; O'GRADY, E.; STAVRIC, B. Lipid content and composition of coffee brews prepared by different methods. Food and Chemical Toxicology, v. 31, n. 34, p. 263-269, 1993. Disponível em: https://doi.org/10.1016/0278- 
SALES, A. L.; PAULA, J.; SILVA, C. M.; CRUZ, A.; MIGUEL, M. A. L.; FARAH, A. Effects of regular and decaffeinated roasted coffee (Coffea arabica and Coffea canephora) extracts and bioactive compounds on in vitro probiotic bacterial growth. Food \& Function, v.11, p. 14101424, 2020. Disponível em: https://doi.org/10.1039/c9fo02589h. Acesso em: 20 dez. 2020.

SANTOS, F. S.; JÚNIOR, L. S. S. Estudo das características físico-químicas de diversos métodos de preparo de café das variedades arábica (Coffea arabica L.) E robusta (Coffea canephora). In: XII Seminário de Iniciação Científica, Feira de Santana, Bahia. Anais do Seminário de Iniciação Científica, 2018 Disponível em: http://dx.doi.org/10.13102/semic.v0i22.4183. Acesso em: 15 jan. 2021.

SANTOS, W. W. V.; ELIAS, A. M. T.; DONATO, M. V. L. C.; MEDEIROS, A. L. T.; BARROS, D. N.; SILVA, M. E. S.; SILVA, S. P. Influência das condições de torra e do processo extrativo em blends de café. Brazilian Journal of Development, v. 6, n. 5, p. 25079 -25092, 2020. Disponível: https://doi.org/10.34117/bjdv6n5-093. Acesso em: 29 dez. 2020.

SUNARHARUM, W. B.; FARHAN, M. Effect of manual brewing techniques on the sensory profiles of Arabica coffees (Aceh Gayo wine process and Bali Kintamani honey process). In: IV International Conference on Agriculture, Environment and Food Security, 2020, Medan Indonesia, p. 1 - 8. Anais do International Conference on Agriculture, Environment and Food Security, 2020. Disponível em: https://iopscience.iop.org/article/10.1088/17551315/454/1/012099. Acesso em: 19 jan. 2021.

TAN, S. A.; SAN, T. H.; YEE, Y. X.; ROSARIOR, V. L.; AGARWAL, T.; SON, Y. C. Antibacterial and Antivirulence Properties of Phenolic Rich Drip Brewed Coffees. In: X International Conference on Biomedical Engineering and Technology, 2020, Tóquio, Japão, p. 46 - 51. Anais do International Conference on Biomedical Engineering and Technology, 2020. Disponível em: https://doi.org/10.1145/3397391.3397430. Acesso em: 05 jan. 2020.

TAVARES, M. P. F.; MOURAD, A. L. Coffee beverage preparation by different methods from an environmental perspective. The International Journal of Life Cycle Assessment, v. 25, p. 1356-1367, 2020. Disponível em: https://doi.org/10.1007/s11367-019-01719-2. Acesso em: 19 jan. 2021.

ZAPATA, A. M. O.; ARANGO, F. O. D.; ROJANO, B. A. THE EFFECT OF GRAVITYDRIP FILTRATION METHODS ON THE CHEMICAL AND SENSORIAL PROPERTIES OF COFFEE (Coffea arabica L. var. Castillo). Coffee Science, v. 14, n. 3, p. 415 - 426, 2019a. Disponível em: https://doi.org/10.25186/cs.v14i3.1603. Acesso em: 01 jan. 2021.

ZAPATA, A. M. O.; ARANGO, F. O. D.; ROJANO, B. A. The effect of pressure filtration coffee preparation methods (Coffea arabica L. var. Castillo) on antioxidant content and activity, and beverage acceptance. DYNA, v. 86, n. 209, p. 261-270, 2019b. Disponível em: http://doi.org/10.15446/dyna.v86n209.75839. Acesso em: 19 jan. 2021. 